\title{
Management of myxoid liposarcoma of the extremity (Review)
}

\author{
YEHIA TFAYLI $^{1}$, AHMAD BAYDOUN $^{2}$, AHMAD SALAHEDDINE NAJA ${ }^{1}$ and SAID SAGHIEH ${ }^{1}$ \\ ${ }^{1}$ Division of Orthopedic Surgery, Department of Surgery, American University of Beirut Medical Center; \\ ${ }^{2}$ Department of Surgery, Saint George Hospital University Medical Center, University of Balamand, 11-0236 Beirut, Lebanon
}

Received January 21, 2021; Accepted April 15, 2021

DOI: 10.3892/ol.2021.12857

\begin{abstract}
Myxoid liposarcoma (MLPS) is the second most common type of LPS after the well differentiated LPS. MLPS is primarily localized to the extremities. The incidence of LPS is $\sim 2$ per million worldwide. MLPS accounts for $\sim 30 \%$ of all LPS cases. MLPS is usually encountered in adults, but can also occur in younger individuals more than other types of LPS. MLPS can be divided into low- and high-grade subtypes, which present with differences in patient prognosis and outcome. Methods of tumor management include surgery, radiotherapy and chemotherapy; however, there is no unified treatment based on tumor characteristics alone. The present manuscript reviews the surgical management, radiotherapeutic and chemotherapeutic approaches reported in the literature for different types of MLPS in the extremities, as well as the post-treatment outcomes. In addition, the present review provides an evidence-based management plan for MLPS in the form of an organogram based on specific tumor and patient parameters.
\end{abstract}

\section{Contents}

1. Introduction

2. Search methodology

3. Study compilation and algorithm

4. Prognostic factors of MLPS

5. Surgical treatment in MLPS

6. RT in MLPS

Correspondence to: Dr Said Saghieh, Division of Orthopedic Surgery, Department of Surgery, American University of Beirut Medical Center, Cairo Street, Riad El-Solh, 11-0236 Beirut, Lebanon E-mail: ss15@aub.edu.lb

Abbreviations: MLPS, myxoid liposarcoma; STS, soft tissue sarcoma; RC, round cell; RT, radiotherapy; LR, local recurrence; OS, overall survival; DFS, disease-free survival; DSS, disease-specific survival; R0, wide local resection; R1, marginal resection; R2, intralesional resection; FNAB, fine needle aspiration biopsy; RCC, RC component; NCCN, National Comprehensive Cancer Network

Key words: algorithm, cancer treatment, RT, sarcoma, surgery
7. Chemotherapy in MLPS

8. Discussion

\section{Introduction}

Myxoid liposarcoma (MLPS) is the second most common subtype of LPS, accounting for $30 \%$ of all cases of LPS and $10 \%$ of all soft tissue sarcomas (STSs) (1). MLPS is most frequently localized to the deep tissues of the thigh (2), and has a distinct morphology, clinical course, molecular markers and chromosomal translocation. MLPS can be divided into two subgroups: i) Low grade, also known as pure MLPS; and ii) high grade, also known as round cell (RC) LPS (3). Differentiation between these two subtypes relies on cellular framework with an accepted cut-off of $5 \%$ for the universally accepted RC component (RCC) (4). Previous studies have shown that the percentage of the hypercellular component is strongly associated with impaired outcome, higher incidence of distant metastasis and poorer prognosis, but with no differentiation by management between the two subtypes $(5,6)$.

Surgical resection with adjuvant radiotherapy (RT) and possible chemotherapy is the primary management option for MLPS (5,7). Several studies have investigated the clinical management outcomes of MLPS, but could not identify a single, unified strategy $(5,7)$. Lack of a well-defined protocol increases the risk of local and distant relapses, negatively impacting patient overall survival (OS) (6). The aim of the present study was to establish an updated algorithm for the multidisciplinary management of MLPS, addressing the roles of surgical resection, chemotherapy and RT in optimizing overall patient prognosis. The study encompasses an individualized approach incorporating demographic, prognostic and specific tumor prognostic parameters.

\section{Search methodology}

Search strategy. Research engine search was conducted on the second week of January 2021 using the PubMed (https://pubmed.ncbi.nlm.nih.gov/), Medline (https://ovidsp. dc2.ovid.com/ovid-a/ovidweb.cgi), Scopus (https://www. scopus.com/search/form.uri?display=basic\#basic) and Google Scholar databases (https://scholar.google.com/). The medical subject headings (MeSH) and terms used at the start of our search strategy were as follows: 'Myxoid liposarcoma', 
'liposarcoma', 'prognostic factors', 'adjuvant chemotherapy', 'adjuvant radiotherapy', 'postoperative radiotherapy' and 'round cell myxoid liposarcoma'. For further information regarding current trials, the ClinicalTrials.gov Registry Platform (https://clinicaltrials.gov/ct2/home) was searched using the following three MeSH terms: 'Myxoid liposarcoma', 'adjuvant radiotherapy', 'liposarcoma' and 'chemotherapy'. Randomized and non-randomized trials, observational studies and case studies were included. Search language was restricted to English.

Inclusion and exclusion criteria. The retrieved databases were independently screened starting with the title, then the abstract; finally, full-text articles were reviewed. Studies about MLPS alone and different types of LPS (including the subtype MLPS) were included. The studies mainly tackled pathophysiology, histology, prognostic parameters and treatment modalities (surgical and non-surgical). Studies not involving MLPS, review articles, studies performed on animals, studies on the molecular aspect of MLPS, studies on MLPS of the trunk only, studies of retroperitoneal MLPS, non-English written studies and Abstracts were excluded. The data were extracted and summarized in a uniform excel sheet format. The excel sheet consisted mainly of the author, years, numbers of cases, treatment, follow-ups, outcomes and conclusion. The information from the retrieved studies that was not relevant to the present study was not added to the excel sheet. The current review aimed to develop an integrated algorithm that combined the available extracted literature focusing on the treatment modality outcomes and the prognostic values to help guide toward favoring a treatment over another.

Quality assessment. The risks of bias were assessed independently by two of the authors. A revised tool to assess risk of bias in randomized clinical trials (RCTs), known as the risk of bias 2 (2019 version) was used for RCT risk of bias (8). The updated version of the tool is structured into six domains accompanied by signaling questions. The six domains were as follows: i) Bias arising from the randomization process; ii) bias due to deviations from intended interventions (effect of assignment to intervention); iii) bias due to deviations from intended interventions (effect of adhering to intervention); iv) bias due to missing outcome data; v) bias in measurement of the outcome; and vi) bias in selection of the reported result. Each item was recorded as 'high risk', 'low risk' or 'some concern risk'.

The 'Newcastle-Ottawa Quality Assessment Form for Cohort Studies' was used for quality assessment of the included cohort studies (9). The quality of each study was further graded as 'good', 'fair' or 'poor' according to the points it received in different components.

The included articles were critically reviewed by the authors of the present review, who are orthopedic surgeons and orthopedic oncologists for basic assessment of confidence levels of each article. Confidence level was not assigned specifically, but only those assigned to a high quality study relevant to the subject were considered for recommendation and analysis. Any inconsistencies were managed through discussions among authors.

\section{Study compilation and algorithm}

A total of 3 RCTs, 1 ongoing RCT, 5 prospective studies (1 randomized), 1 case study and 27 retrospective studies were selected (Table SI). The main stratification of the algorithm was based on the size of the tumor $(<5,5-10$ and $>10 \mathrm{~cm})$. The second stratification was based on the depth of the tumor [superficial (histology, type of resection and location) for further adjuvant or neoadjuvant treatment if needed, or deep]. Each patient was then stratified according to its demographical and tumor characteristics.

\section{Prognostic factors of MLPS}

Age. Multiple prognostic factors influence the OS and cancer-specific survival of patients with MLPS (10). Historically, age has been considered a major attributor to the overall natural progression of MLPS, and thus stratification of management should be conducted based on age cut-offs for optimization of care with the most effective modality. Wu et al (11) demonstrated that increased age was associated with poorer OS and cancer-specific survival in patients with MLPS. An age of $>30$ years was shown to be an independent risk factor for poorer OS, and patients $>60$ years of age had a worse prognosis than those $>30$ years old (11). Another study indicated that an age of $>60$ years was an independent predictor of worse OS and disease-free survival (DFS) for extremity and trunk MLPS (12). In addition, an epidemiological study (13) on the prognostic factors of MLPS revealed that patients $>40$ years of age had a higher probability of developing RC tumors, which was itself a predictor of worse DFS. Although a specific age was not identified, elderly patients had a higher tendency to acquire MLPS with a greater percentage of RC tumors (13). In a study of the effects of age and histology on operable LPS, Greto et al (14) found that patients $>65$ years of age had a higher risk of high grade histology and local recurrence (LR) over a follow-up period of 8.6 years. In a study of 95 patients with MLPS with or without RC differentiation, an age of $>45$ years, tumor size of $>10 \mathrm{~cm}$ (significant only with univariate analysis) and tumor necrosis were all found to be associated with a worse OS (15). The hypothetical suggestions behind age and poor prognosis are a decrease in patient immunity and inferior host DNA repair mechanisms, which result in a higher probability of mismatch errors, and cause oncogene activation and/or amplification or defects in tumor suppressor genes (16). A study conducted at the University of Groningen Medical Center revealed that patients $>45$ years old had the worst prognosis (17). Dürr et al (10) found that patients with MLPS aged between 45 and 60 years had a worse prognosis for OS. This finding was reinforced by multivariate analysis in a number of other studies $(15,18,19)$. A nomogram of 2,163 patients with STS was constructed by the Memorial Sloan-Kettering Cancer Center to analyze the disease-specific survival (DSS) of common types of STS (20). The major predictors of survival were listed, including age, and it was demonstrated that patients $>65$ years old had a $6 \%$ reduction of 8 years DSS compared with those $>45$ years old (18). On the other hand, a study conducted at the Cleveland Clinic revealed no significant difference in prognosis between patients older or younger than 45 years of age (19). Additionally, various 
studies conducted in the early 1990s have shown that age at diagnosis has no prognostic value $(21,22)$. The median age of MLPS presentation is 45 years, and most studies have selected 45 years as the cut-off value to assess the prognosis of patients with MLPS, primarily since it provides the strongest statistical power (23). However, when 45-year-old patients were compared with those between 60 and 65 years old (as is the case in the majority of reported studies), it was well established that a higher age was associated with a poorer prognosis $(14,15)$. Thus, age is as an impactful influencing factor for patients with MLPS. As such, in the present study, an organogram or algorithm was constructed using an age of $>65$ years ( \pm 5 years) as the cut-off for the need for any additional or more aggressive therapeutic intervention (Figs. 1-3), in view of its negative prognostic value.

Histology. Multiple subtypes of LPS exist, including pleomorphic, well-differentiated myxoid and RC. RC is the less differentiated form of MLPS, and both can be frequently found in the same tumor (19). Fiore et al (6) studied the prognostic factors of multiple subtypes of MLPS, and found that RCLPS (which is graded as type II or III in contrast to pure MLPS, also known as grade I) exhibited more aggressive behavior, with a $22 \%$ incidence of distant metastasis, compared with $5 \%$ in primary MLPS. In a retrospective study of 49 patients on the clinicopathological prognostic factors of MLPS, ten Heuvel et al (17) established that patents with MLPS and $>5 \%$ RCC had a higher tendency to develop metastasis, with an associated poorer survival $(\mathrm{P}=0.0004$ and $\mathrm{P}=0.03$ from univariate and multivariate analysis, respectively). This finding was further reinforced by Antonescu et al (18) $(\mathrm{P}=0.01$ and $\mathrm{P}=0.02$ from univariate and multivariate analysis, respectively). Furthermore, Smith et al (19) revealed that $>5 \% \mathrm{RCC}$ is a factor for poorer prognosis in univariate analysis only $(\mathrm{P}<0.05)$. In the Mayo Clinic study, $35 \%$ of the 14 patients with MLPS $(5-25 \%$ RCC) developed metastasis, and $29 \%$ died from the disease, although this finding was not statistically significant (15). For patients with $>25 \%$ RCC, $58 \%$ succumbed to associated metastases and 54\% died from causes associated with the primary tumor, which was statistically significantly in both univariate and multivariate analyses (15). Evans (24) observed a similar finding regarding the effect of MLPS with $25 \% \mathrm{RC}$ on OS, although the sample size was small. Patients with pure RCMLPS have a poorer prognosis than those with pure MLPS. Several cut-offs have been listed to identify which range of RCC would be the best attributor of overall prognosis (as stated in the aforementioned studies) $(15,17,18)$. Nevertheless, other studies have stated that any RC component is associated with poorer outcome $(3,17,19,25,26)$. For example, Haniball et al (4) investigated the prognostic factors associated with MLPS, and demonstrated that a higher RCC predicts a worse survival outcome. Thus, it was concluded that patients with $>5 \%$ RCC should be considered for adjuvant RT and/or chemotherapy $(4,27)$. Histological prognosis of RCMLPS was also demonstrated by Bartlett et al (28), regarding its impact on survival and LR. In addition, the importance of adjuvant RT was further elucidated by Yang et al (29), Pisters et al (30) and Harrison et al (31), who determined that high-grade LPS (i.e. RC) was highly affected by adjuvant RT, which prevents LR post-limb salvage surgery. However, 5\% RCC was selected as a cut-off for more aggressive surgical and non-surgical treatment in the present study, as shown in Fig. 1. In addition, since grading is the most impactful factor of prognosis compared with size, age and location (as indicated by the nomogram constructed by the Memorial Sloan-Kettering Cancer Center) (20), the safest surgical and non-surgical treatment was selected where applicable to decrease the risk of LR where possible (32). Nevertheless, it is important to mention that this nomogram was constructed for all histological LPSs, including MLPS.

Size. The average size of MLPS is $\sim 8-12 \mathrm{~cm}$, with a range between 1.5 and $25 \mathrm{~cm}$ (10). Several studies have discussed the prognostic value of tumor size in patients with MLPS. Orson et al (33) and Reitan et al (34) both demonstrated that larger tumors were associated with poorer prognosis. Moreover, four larger studies $(7,15,18,20)$ assessed several prognostic factors for MLPS with varying results. One study showed that tumors $>5 \mathrm{~cm}$ were associated with worse DSS compared with those $<5 \mathrm{~cm}$ (17). Univariate analysis revealed that tumors $>10 \mathrm{~cm}$ indicated lower OS rates compared with patients with smaller tumors (15); however, this finding was not statistically significant for multivariable analysis. Zheng et al (7) showed that the greater the diameter of the tumor, the lower the OS rate, and the higher the risk of metastasis. A similar result has been demonstrated in various other studies illustrating the impact of tumor size on prognosis (28,35-38). For example, in a study of $>3,752$ patients with LPS, Callegaro et al (20) demonstrated that a tumor size of $11 \mathrm{~cm}$ was associated with worse 5-year survival rate than that of patients with $4-\mathrm{cm}$ tumors, and that patients aged $>69$ years had a worse 5-year survival rate than those $>42$ years old; these findings were both statistically significant. Notably, the aforementioned studies included various histological types of LPS, including MLPS. On the other hand, a study conducted by Smith et al (19) demonstrated that size did not affect the clinical outcomes of patients with MLPS and Nishida et al (12) stated that depth and size were not independent prognostic factors for survival. In a study by Dürr et al (10), the mean size of the MLPS was $12 \mathrm{~cm}$. It was demonstrated that tumor size had a significant prognostic value in terms of OS, and that RT decreased the size of the tumor, but did not affect LR (10). These findings are in contrast to those of Guadagnolo et al (39), who showed a significant decrease in LR. Differentiating size cut-offs has several advantages in MLPS. Although the literature revealed no real consensus on which tumor sizes are associated with the worst prognostic outcome, most studies have used cut-offs of $<5,5-10$ and $>10 \mathrm{~cm}$. It is important to note that larger tumors have a greater probability of being closer to essential structures, which may jeopardize the extent of resection in limb salvage surgery. Thus, the current review suggests that when wide surgical resection (R0) is not possible, the only alternative is either marginal (R1) or intralesional (R2) resection, which increases the risk of recurrence with or without affecting survival. Therefore, it is imperative to consider tumor size when treating patients with MLPS, as it may guide clinicians to the appropriate timely management for optimization of cancer-free survival, while minimizing the risk to vital structures. 


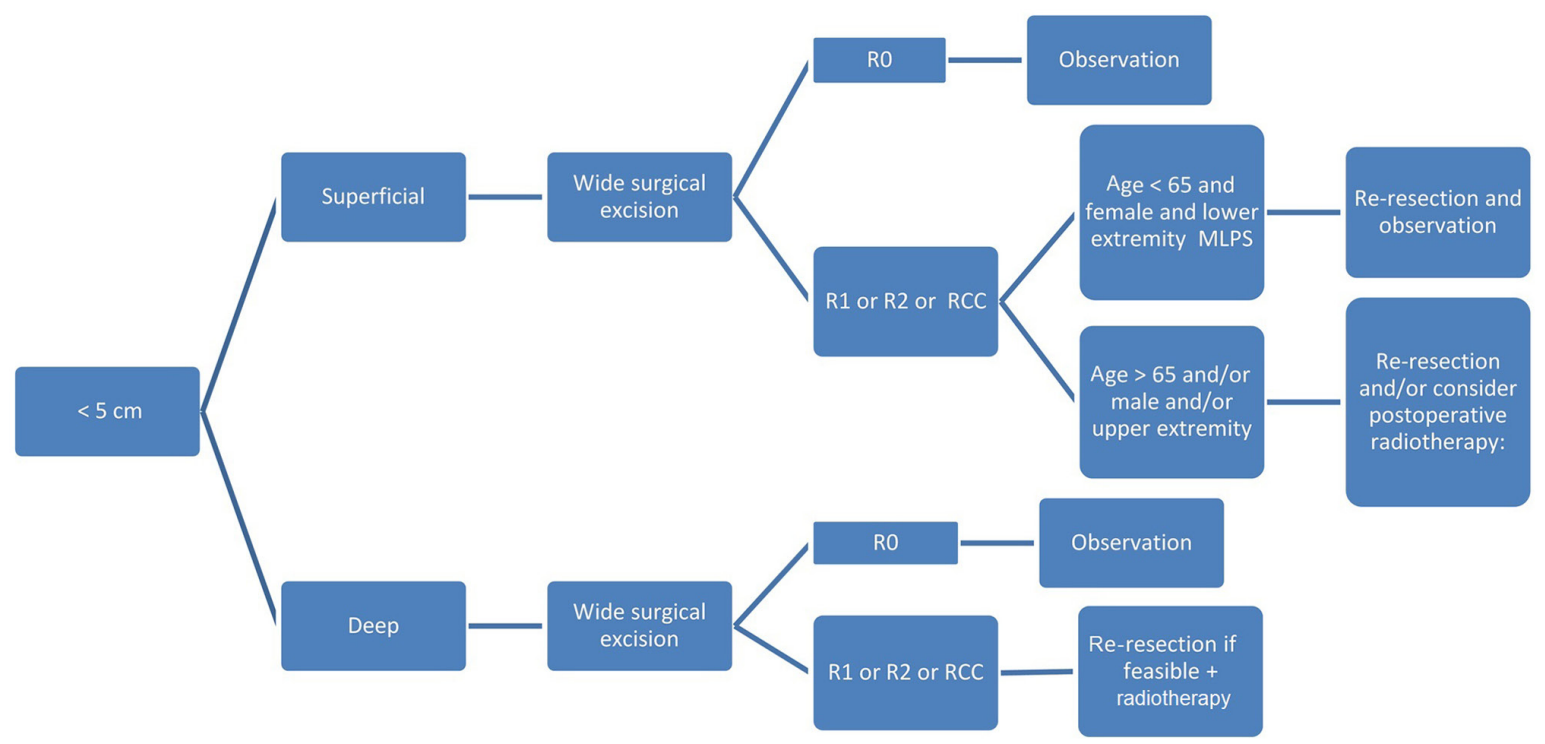

Figure 1. Management algorithm for MLPS $<5 \mathrm{~cm}$ in size. Its management includes wide resection, but the margin of resection decides the next step in management. R0, wide local resection; R1, marginal resection; R2, intralesional resection; MLPS, myxoid liposarcoma; RCC, round cell component.

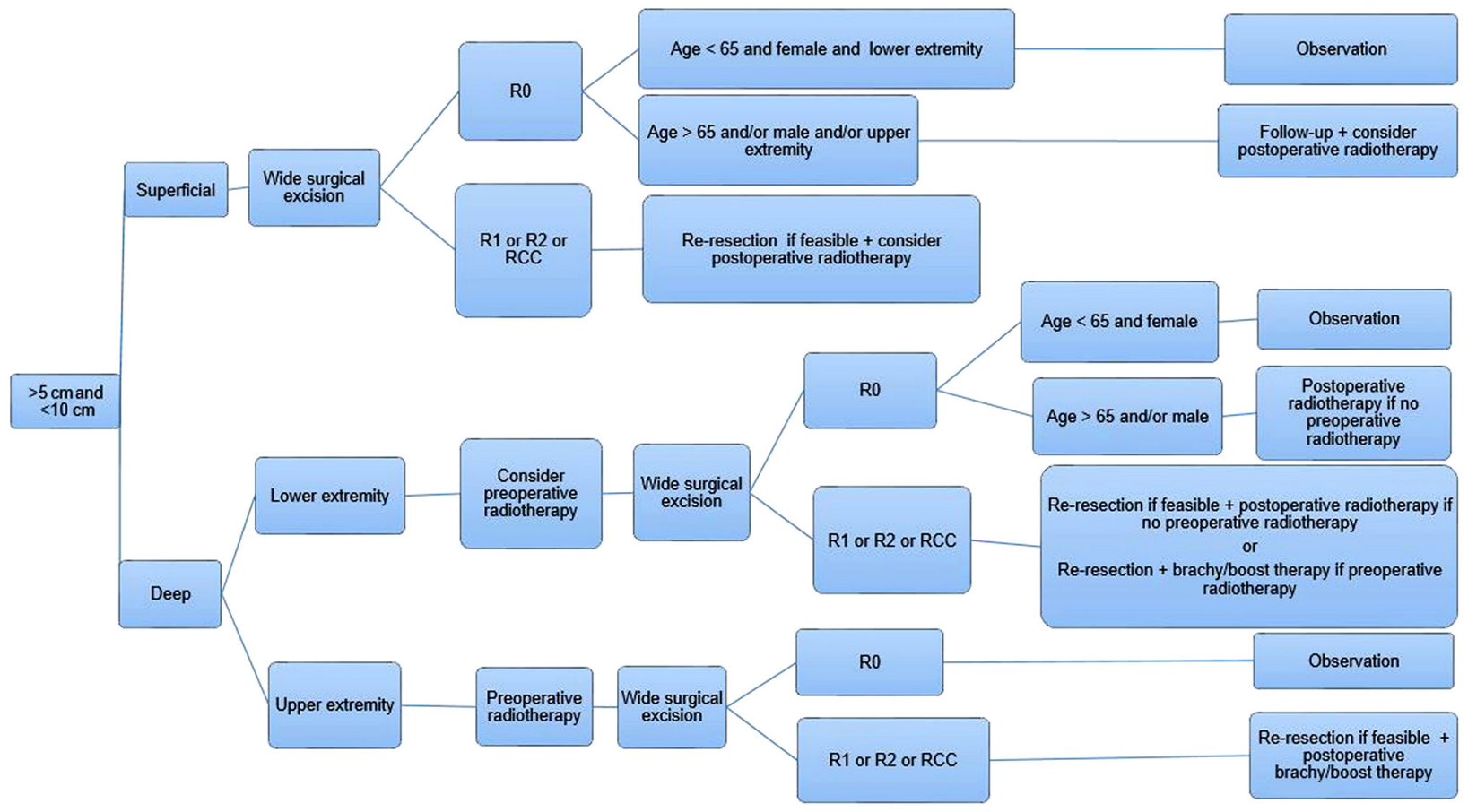

Figure 2. Management algorithm for myxoid liposarcoma between 5 and $10 \mathrm{~cm}$ in size. Superficial tumors will need wide resection, while deep tumors will require radiotherapy prior to resection. R0, wide local resection; R1, marginal resection; R2, intralesional resection; RCC, round cell component.

Location, depth and sex. Various studies have identified that trunk LPS is associated with a poorer outcome than extremity LPS $(12,19,40)$. However, the difference in survival and prognosis between tumors of the upper and lower extremities is not commonly discussed in literature. No significant difference was observed by Wu et al (11) when comparing upper and lower extremity LPS, though univariate analysis revealed a lower OS in patients with lower extremity LPS. Lansu et al (13) demonstrated that upper extremity LPS was associated with a poorer OS $(\mathrm{P}=0.00001)$ in patients when followed-up over 7.6 years. Muratori et al (41) found that metastasis-free survival for lower extremity tumors was higher compared with that of the upper extremities, although without statistical significance. Nishida et al (12) studied the role of location and depth in terms of OS and DFS, with a follow-up period of 12 months. The OS and DFS for upper and lower extremity MLPS were 80, 69, 93 and $81 \%$, respectively, but did not reach statistical significance (12). A possible explanation for this may be the smaller surface area of the upper compared with the lower extremity, and thus, there is less space for the tumor to increase in size without affecting vital neurovascular structures. In addition, due to the crowded anatomy of the upper extremity in terms of 


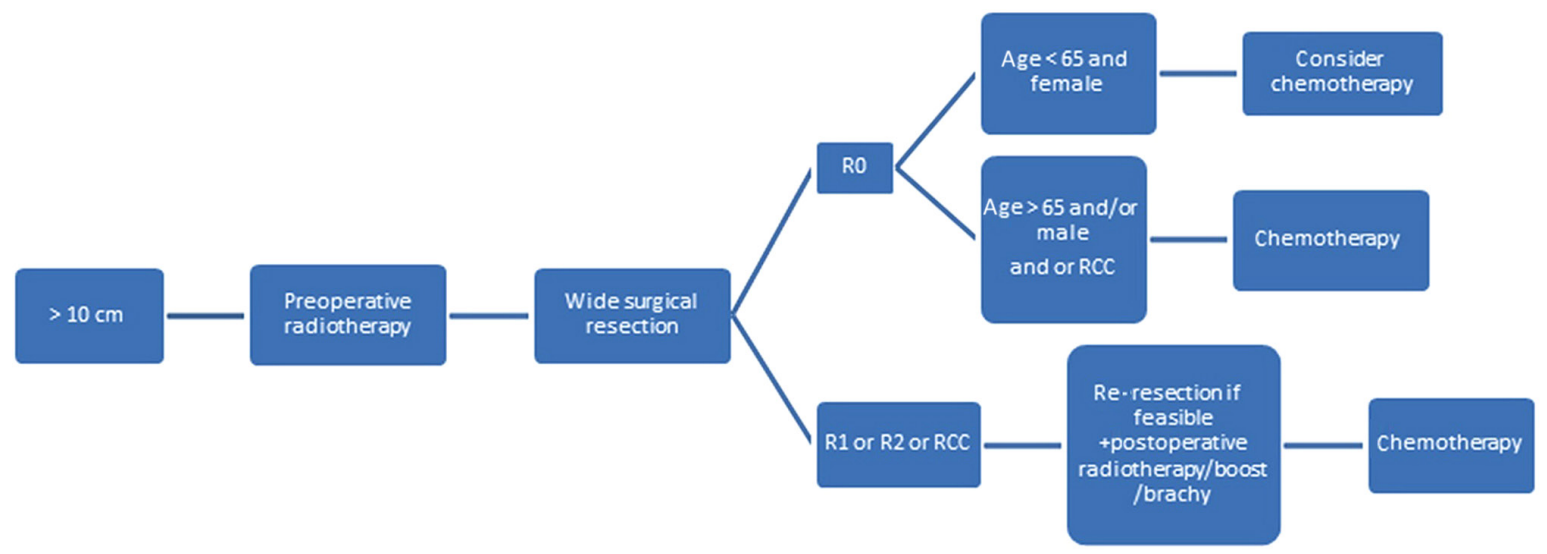

Figure 3. Management algorithm for myxoid liposarcoma $>10 \mathrm{~cm}$ in size. All these tumors should be managed by radiotherapy followed by resection. Further steps depend on the margin of resection, age and sex of the patient. R0, wide local resection; R1, marginal resection; R2, intralesional resection; RCC, round cell component.

compartments and neurovascular structures, the probability of complete resection of the tumor is decreased compared with that of the lower extremity, assuming the same lesion size (12).

In terms of depth, patients with deep tumors experienced 85 and $70 \%$ OS and DFS rates, respectively, and 100 and $78 \%$ OS and DFS rates for superficial tumors, respectively, but without statistical significance $(12,20)$. In a study of LPS, Gronchi et al (42) revealed that deep tumors were associated with worse DFS and OS times compared with superficial tumors. A similar finding was observed by Greto et al (14) surrounding the prognosis of deep compared with superficial tumors. Aiba et al (43) did not reveal an association between depth and histological response in patients with MLPS, and Bartlett et al (28) demonstrated that deep tumors were associated with a higher risk of LR (without statistical significance) compared with superficial tumors, during a 5.4-year follow-up period.

Male patients have been shown to have significantly worse survival compared with female patients with MLPS (11). Vos et al (44) demonstrated that male sex was the only significant factor for the risk of LR in patients with LPS $(\mathrm{P}=0.037)$. The same prognostic impact of male sex was further reinforced by Toulmonde et al (45); however the study was conducted on patients with retroperitoneal MLPS. By contrast, Salduz et al (46) did not reveal any effect of sex on the prognosis of patients with MLPS. This evidence-based literature guided the selection of more aggressive treatment post-surgical resection in male patients, or patients with upper extremity or deep tumors in the present study, as is apparent from the resulting organograms (Figs. 1-3).

\section{Surgical treatment in MLPS}

Surgical resection remains the first-line treatment for almost all cases of STS, including primary MLPS of the extremities, with the exception of some diffusely metastatic conditions $(7,47,48)$. Historically, amputation was once considered the standard therapy to attain local control in patients with extremity STS (49). However, improvements in imaging, implementation of adjuvant therapy and technical advances in reconstructive surgical procedures have decreased the requirement for amputation. In 1982, a randomized control study of 43 patients conducted by Rosenberg et al (50) showed that limb-sparing surgery with RT was an effective treatment in patients with high-grade STS of the extremities, with a LR rate of $15 \%$, and no difference in OS and DFS rate compared with amputation. Limb salvage surgery in MLPS is beneficial for the following reasons: i) The low potential for local invasion and distant metastasis compared with other STSs; and ii) its high radiosensitivity, insuring adequate preoperative shrinkage of the tumor, and securing margin-free surgical resection while minimizing the risk of recurrence $(3,51)$. The challenge to surgeons resecting soft tissue tumors is removing the mass with a sufficient margin of surrounding normal tissue, while maximizing postoperative physical function (52). Detailed surgical approaches and techniques for resecting STSs are beyond the scope of this article. However, MLPS resection is broadly divided into R0 and R1 (53). In very rare cases, the surgeon may fail to completely resect the tumor, primarily due to adherence to neurovascular bundles. In these cases, it is considered to be R2. R0 is accomplished through resection of the lesion, its pseudocapsule and/or reactive zone with a surrounding of completely normal tissue, but without removing the entire compartment. The cut-off margin of normal tissue varies between 1 and $2 \mathrm{~cm}$, or if the fascial plane is intact (53). On the other hand, R1 is achieved by also removing the lesion as aforementioned; however, the plane of dissection is through the pseudocapsule, and thus, may potentially retain microscopic tissue portions at the margin of the wound (53).

The importance of R0 has been demonstrated by Zheng et al (7). In their study, MLPS with R0 had no LR compared with $61 \% \mathrm{LR}$ in patients with R1 (7). The average follow-up period for patients with R0 was 43.7 months, and $60.9 \%$ of patients who underwent R1 experienced LR, with an average follow-up period of 75 month. Nevertheless, no significant difference was observed between the different surgical modalities (7). Notably, patients who underwent R1 and R0 had a tumor size of $17.2 \pm 8.8$ and $8.6 \pm 4.7 \mathrm{~cm}$, respectively, with a significant difference in tumor size; of the patients who underwent R0, 5 received adjuvant chemotherapy or RT, and 3 succumbed to the disease (7). Furthermore, Dürr et al (10) 
observed the same finding with no recurrences following R0 compared with $33 \%$ after R1, despite the use of RT and chemotherapy in the majority of patients. A retrospective study by Nishida et al (12) (with an 8-year follow-up period) revealed that MLPS with R0 could be locally controlled with surgery alone, without the need for RT. No decrease in LR rate was observed in the minority of patients treated with RT. Moreover, no significant advantage was observed with RT to prevent LR in patients with negative margins (12). Haniball et al (4) did not obtain promising results from postoperative RT in patients with R1 or R2. The risk of LR in this study was found to be strictly associated with the margins and the presence of $>5 \%$ $\mathrm{RCC}$, irrespective of whether the patients received RT or not (4).

\section{6. $R T$ in MLPS}

The primary characteristic of MLPS, compared with other types of STS, is high radiosensitivity (54). RT can be used either preoperatively or postoperatively (55), and preoperative RT has gained popularity in STS. The main advantages of preoperative RT are reduction in the field and dose, as well as potential tumor shrinkage. In 2004, Pitson et al (56) highlighted a superior preoperative response to RT in patients with MLPS compared with that for other subtypes of STS, and there was a significantly greater reduction in tumor size when treated with RT compared with undifferentiated STS. In 2007, Engström et al (36) reported a marked decrease in MLPS volume following preoperative RT; 23 out of 30 irradiated tumors showed a median reduction in volume of $52 \%$, and it was postulated that RT induced the histopathological accumulation of mature lipoma-like areas and tumor volume reduction that may facilitate resectability. No LR was observed in patients with MLPS who had received preoperative RT, as demonstrated by Chung et al (51). Only one instance of LR was reported by Salduz et al (46) in a study of 23 patients with MLPS treated with preoperative RT. Finally, Moreau et al (3) concluded that RT prevented local relapse $(\mathrm{P}<0.001)$ and also induced a 7-fold decrease in the 5-year LR rate of patients with positive margins $(\mathrm{P}<0.05)$.

Moreover, Chowdhry et al (57) observed differences between patients who had received preoperative RT (LR 3\%) compared with those who underwent postoperative RT (LR 11\%). Since 1979, preoperative RT combined with conservative surgery has been the standard treatment for patients with MLPS, with no observable LR (39). However, the aforementioned study also included patients referred to their center following resection, thus undergoing preoperative RT was not always an option. In these patients, postoperative RT was associated with $94 \%$ local control despite the fact that $29 \%$ patients received R1 or undetermined margin resection (39). Fiore et al (6) reported on mixed groups of patients where some received perioperative RT and others underwent surgery alone, and multivariate analysis revealed that postoperative RT was associated with a lower LR rate. Furthermore, ten Heuvel et al (17) identified a significant decrease in LR when surgery was coupled with postoperative RT ( $8 \%$ vs. $44 \%$; $\mathrm{P}=0.01$ ). However, the most convincing argument for RT was demonstrated by Hatano et al (58), who showed that MLPS treated by R1 or R2, combined with RT, resulted in $100 \%$ local control in 10 patients over a follow-up period of 58 months. The choice between using RT as a preoperative or postoperative modality in MLPS is still under debate. However, the aforementioned findings on preoperative RT in STS, including MLPS, have shown it to be an extensively promising modality for the management of MLPS. The reasons for this include a reduction in late toxicity, improved outcome, maintaining adequate local control and allowing borderline tumors to become surgically accessible $(38,59)$. Le Grange et al (38) showed that preoperative RT resulted in a marked decrease in the volume of soft tissue tumors, which was most apparent in MLPS. Preoperative RT was shown to be an effective treatment, primarily for high-risk borderline tumors, justifying its importance as the modality of choice in RCMLPS due to its high-grade features, risk of recurrence, large deep features and its proximity to sensitive locations (38). Notably, tumor volume reduction in patients who underwent preoperative RT was inversely proportional to tumor grade (38). Postoperative RT has been associated with a higher risk of fibrosis, joint stiffness and marginally predictive edema compared with preoperative RT (59). In addition, preoperative RT allows a lower threshold for high-dose postoperative RT, which decreases the risk of dose-associated radiation toxicity (59). The radiosensitivity of MLPS, including RCMLPS, was shown by a reduction in tumor size, as well as an increasing percentage of hyalinization, intra-mural fat and necrosis, enabling its use for the optimization and facilitation of surgical resection when used preoperatively (60). In $92 \%$ of cases, preoperative RT decreased MLPS size by an average of $25-30 \%$ in tumors of $12 \mathrm{~cm}$ at 48 days post treatment initiation, thus shrinking the tumor to $\sim 8 \mathrm{~cm}$ with a hyalinization content of almost $100 \%$ (60). Notably, $7 / 13$ patients in the study also received preoperative chemotherapy (60). Roberge et al (61) demonstrated an $82 \%$ median decrease in tumor volume following external beam RT. This radiosensitivity was primarily associated with damage to the medium-sized arterioles (62). Although Eilber and Eckardt (48) demonstrated that necrotic content post-RT was associated with a decreased risk of recurrence and improved survival, this finding was not proven to be accurate according to Wortman et al (60) and Mullen et al (63).

The recent DOREMY trial (NCT02106312) is investigating the possibility of an RT dose reduction for MLPS without jeopardizing clinical outcomes (https://clinicaltrials.gov/ct2/show/NCT02106312). Through their phase II results (13) it was revealed that a 36-Gy dose delivered in once-daily 2-Gy fractions resulted in the same clinical outcome with a possible decrease in toxicity. These findings reinforce the role of preoperative RT in MLPS where adequate surgical resection is unachievable or where the tumor is inoperable $(13,36)$. Postoperative RT was once more commonly used than preoperative RT for STSs (64). However, since 2004, there has been a steady increase in the use of preoperative RT. Postoperative RT showed no superior benefit in terms of survival and LR compared with preoperative RT (64). In addition, local control is better achieved using preoperative RT (65). Overall, the timing of use for each modality is multifactorial, and should be evaluated on a case-by-case basis. It is safe to conclude that postoperative RT may be used as an adjunct treatment for use in patients with small tumors 
$(<10 \mathrm{~cm})$ where the true depth and approximation to vital structures is underestimated, leading to non-optimal resection. Nevertheless, due to its high dose, higher late morbidity is expected in postoperative RT, but a higher rate of infection is seen in preoperative RT (66). Currently, the standard treatment for STS management is external beam intensity modulated RT due to its superior local control compared with brachytherapy (67). The majority of studies $(25,35,42,43,64)$ comparing preoperative and postoperative RT include all STS subtypes, not just MLPS. However, to the best of our knowledge, high-quality evidence solely addressing comparative studies of RT timing in MLPS is lacking.

\section{Chemotherapy in MLPS}

The role of chemotherapy in patients with MLPS has been discussed from several perspectives: i) As a neoadjuvant for local control of primary MLPS; ii) as an adjuvant in a postoperative setting; and iii) for the treatment of metastatic MLPS. Adjuvant chemotherapy in STS (including MLPS) has been studied in multiple randomized control trials in the 1990s, with a meta-analysis published in The Lancet, concluding that doxorubicin was associated with a significant improvement in recurrence-free survival with no associated improvement in OS (68). Another study emphasized the role of ifosfamide to treat primary STS with differing conclusions (2). In 2004, Eilber and Eckardt (48) investigated the effects of both doxorubicin and ifosfomide in patients with high-risk primary STS (including M/RCLPS), demonstrating that ifosfomide was associated with improved DSS compared with patients who did not receive chemotherapy $(\mathrm{P}=0.0003)$. This association was the strongest for tumors $>10 \mathrm{~cm}$, high grade (e.g. RC), primary and extremity LPS. In addition, M/RCLPS was shown to be an independent prognostic factor for improved DSS $(\mathrm{P}=0.03)$ (48). In positive-margin MLPS and post re-excision, complementary RT combined with adjuvant chemotherapy should be implemented depending on the histology, type, extension of the tumor and size $(29,69,70)$.

Previously, neoadjuvant chemotherapy was rarely discussed for the treatment of MLPS. A phase II clinical trial of neoadjuvant trabectedin in patients with advanced localized MLPS demonstrated that $1.5 \mathrm{mg} / \mathrm{m}^{2}$ trabectedin every 3 weeks had significant efficacy and minimal toxicity (42). Tumors with the complete disappearance of malignant tissue were in the lower extremities, with a size between 5 and $10 \mathrm{~cm}$ (42). A study conducted by Guadagnolo et al (39) stated that for patients with tumor size $>10 \mathrm{~cm}$, treatment should be supplemented with neoadjuvant doxorubicin-based therapy. Axitinib has shown promising results for the treatment of MLPS, exerting its anti-angiogenic and anti-tumorigenic effects through the VEGF signaling pathway (71). In unresectable pulmonary metastatic and extrapulmonary metastatic STS, including MLPS and RCLPS, patient prognosis is unfavorable, thus systemic chemotherapy is required, and surgery should be considered as a palliative treatment option (41).

Several clinical trials have investigated the role of combined adjuvant chemotherapy (e.g., trabectidin) and RT in MLPS $(42,51)$. This combination has the potential to decrease RT-associated toxicity, and may also act in a synergistic manner for tumor eradication $(42,72)$. Furthermore, the
TRASTS trial has shown that chemotherapy-RT combination in patients with MLPS is safe, and a potential step towards the delivery of combination therapy $(42,51,72,73)$. It is important to note that although the extent of necrosis post-neoadjuvant chemo-RT in MLPS was not large, the percentage was not associated with the outcome (63). Thus, volume reduction should be the primary focus to reach a tumor size that is appropriately resectable.

\section{Discussion}

MLPS has distinctive features compared with other types of STS. Specific chromosomal translocations have been identified in MLPS, which consist of fusions between the fusion binding protein gene and the $\mathrm{C} / \mathrm{EBP}$ homologous protein (CHOP) gene $[(\mathrm{t} 12 ; 16)(\mathrm{q} 13 ; \mathrm{p} 11)]$ in $90 \%$ of tumors, and between the RNA-binding protein related to Ewing sarcoma and CHOP genes [(t12;22)(q13;q12)] in $>5 \%$ of tumors (17). In difficult cases, PCR detection of these translocations allows for a specific diagnosis of MLPS (15). Among the STSs, fine needle aspiration biopsy (FNAB) is of significant efficacy in adult myxoid STSs, with the majority being diagnosed with a reasonable level of accuracy, based solely on their cytological distinctions $(74,75)$. Nonetheless, FNAB is not very accurate in differentiating subtypes of MLPS, including RCMLPS $(23,74)$. Despite the distinct prognostic factors for OS in the presence of $>5 \%$ RCC, limited data are available that may influence the course of treatment. MLPS and RCLPS were considered as a single entity in the present study, and both have been included in the resulting organograms.

MLPS has a distinctive pattern of extrapulmonary metastases, and screening and follow-ups should include history taking, thorough physical exams and imaging (i.e. whole body MRI and CT scans of the chest, abdomen, pelvis and thorax) (76).

Surgical resection remains the first-line treatment for MLPS. The resection margin is one of the most important factors affecting survival in patients with STS, as observed by Kim et al (77) over a median follow-up period of 48 months. However, due to its high radiosensitivity, few centers advocate more conservative resection in MLPS, and preoperative RT is the preferred type of adjuvant therapy (50). However, chemotherapy may also serve a minor role, particularly in tumors $>10 \mathrm{~cm}$ in size (50).

Nevertheless, the specific prognostic factors and standardized algorithm required to maximize survival rate with the highest quality of life remain limited. Thus, a detailed algorithm with the potential prognostic and therapeutic factors (combining size, grade, location and follow-up) to detect recurrences is required to establish a schema for clinical guidance. Several prognostic factors influence LR rate and OS in MLPS (18), the most important of which are surgical margins, the presence of a RCC and tumor size $(4,12,17)$. The latter is of utmost importance, and serves as a considerable limiting factor in management modification (e.g. the benefit of incorporating adjuvant chemotherapy in tumors $>10 \mathrm{~cm}$ in size) (69). A retrospective study of 34 patients with MLPS with a 65-month mean follow-up period showed that tumor size was an independent risk factor for MLPS metastasis, with an average tumor diameter of $21.7 \mathrm{~cm}$ (7). Another study of 
49 patients with a median follow-up of 101 months demonstrated that tumor grade, size and age negatively impacted survival (17). Haniball et al (4) analyzed the prognostic factors and metastatic patterns in primary M/RCLPS, and discovered that age $>50$ years, size $>10 \mathrm{~cm}$ and $>5 \%$ RCC on histology were significant factors for a poor prognosis $(\mathrm{P}=0.027, \mathrm{P}=0.03$ and $\mathrm{P}=0.0001$, respectively). The study was conducted over a 10-year follow-up period with 130 tumors, including 88 with $<5 \%$ RCC and 42 with $>5 \%$ RCC (4). It was also suggested that adjuvant chemotherapy and RT should be considered for patients with $>5 \%$ RCC (4). In a multicenter retrospective study of 418 cases of MLPS and RCLPS, Moreau et al (3) demonstrated that with a 5.2-year follow-up period, a tumor diameter $>10 \mathrm{~cm}$ was associated with an increased risk of metastasis and death $(\mathrm{P}=0.024)$, and that RT effectively prevented $\mathrm{LR}$, and should therefore be administered as a neoadjuvant. By contrast, Nishida et al (12) concluded that size and depth did not affect patient prognosis in MLPS of the extremities. Thus, the majority of studies suggest that size is a critical factor in the overall prognosis of patients with MLPS. Hence, in the present study, the management organogram was stratified based on size cut-offs of $<5,5-10$ and $>10 \mathrm{~cm}$ (Figs. 1-3).

There is adequate data recommending the use of R0 surgical resection for MLPS, with the aim to achieve negative margins (55). Nevertheless, the definition of negative-margin R0 varies considerably, complicating the comparison between different studies (78-80). In the present study, a 1-cm negative margin was adopted for the algorithm, which was based on the National Comprehensive Cancer Network (NCCN) STS clinical practice guidelines (55). Moreover, intramuscular margins should not receive the same consideration as extra-compartmental margins. Deep fascia, periosteum and fatty tissues around the vascular bundles may act as a barrier, and thus, a few millimeters of healthy tissue are enough to ensure margin-free resection. Nevertheless, intracompartmental margins should be $\geq 1 \mathrm{~cm}$ for the optimization of surgical resection.

For the management of superficial MLPS $<5 \mathrm{~cm}$ (Fig. 1), $\mathrm{R} 0$ excision should be adopted as the surgical procedure of choice. If the surgical margins are disease-free (margins $>1 \mathrm{~cm}$ or an intact fascial plane), observation with frequent follow-ups is required, regardless of age. This is reinforced by multiple studies demonstrating that surgery without RT is the favored treatment type to enable local control, especially if confirmed by negative-margin resection in tumors $<5 \mathrm{~cm}(6,11,12)$. If the surgical margin shows positive microscopic margins (R1), positive macroscopic margins (R2) or if pathology indicates RCMLPS, the next actions are stratified as follows: i) Repeated resection and observation if the patient is <65 years old, female and with lower extremity MLPS; or ii) consider postoperative RT if the patient is $>65$, male or has upper extremity MLPS (Fig. 1).

The analogy behind this management has been demonstrated by several studies $(11-14,18)$, showing that age $>65$ years, female sex and lower extremity MLPS were associated with lower LR-free survival, distant metastasis-free survival, OS, DSS and local control, respectively (6). Age $>65$ years is an independent prognostic factor affecting local and distant recurrence, as well as OS $(12,18)$. In fact, age is a well-defined negative prognostic factor for clinical outcome in patients with MLPS, which can be attributed to associated comorbidities, late tumor presentation and genomic alterations present in older patients (55). Lower control rates on the upper extremity are primarily attributed to the anatomical difficulty in achieving R0 (12), which explains the use of postoperative RT if positive surgical margins are identified. The adopted postoperative RT regimen can be used for high-grade STS alone or low grade tumors with the aforementioned risk factors (NCCN clinical practice guidelines) (55).

If the MLPS size is $<5 \mathrm{~cm}$ (Fig. 1), deep and with negative R0 margins (i.e., wide local resection), the same management as superficial MLPS is recommended, which is post-operative follow-up. This is based on studies $(7,12,20)$ showing that tumor depth is not a prognostic factor, and does not impact cancer-free survival in MLPS. However, if the tumor is deep and with post-operative resection or R2 margins (i.e. intralesional), or if it has an $\mathrm{RCC}$, repeated resection is recommended if feasible, as well as administration of postoperative RT (Fig. 1).

There is currently limited literature surrounding the management of MLPS with a size between 5 and $10 \mathrm{~cm}$. In the current algorithm, surgery with negative-margin resection may be enough to treat superficial tumors, but only in female patients $<65$ years old with lower extremity tumors. Thus, postoperative RT should be considered as an adjuvant treatment in all other patients. If the surgical margins are positive (R1 or R2), or the tumor has an RCC, repeat resection and postoperative RT should be implemented, irrespective of age, sex or tumor location (Fig. 2).

If the tumor is deep and is in the lower extremities, preoperative RT should be considered before R0 surgical excision. In patients who proceed directly to surgery, only female patients $<65$ years old may not require postoperative radiation if $\mathrm{R} 0$ is achieved. When the surgical margins are positive, or if the tumor has an RCC, re-resection and postoperative RT should be implemented, irrespective of age, sex and tumor location. For patients that have received neoadjuvant radiation, brachy/boost therapy should be administered to minimize radiation exposure, while targeting residual margins and limiting the possibility of LR (Fig. 2).

Due to the challenging aspects of resection, deep tumors located in the upper extremities should first be treated with preoperative RT, and then R0 surgical resection. If the surgical margins are positive (R1 or R2) or the tumor has an $\mathrm{RCC}$, then repeated resection should be implemented if possible, as well as postoperative brachy/boost therapy (Fig. 2).

Finally, irrespective of depth and location, MLPSs $>10 \mathrm{~cm}$ in size should be managed with preoperative RT, which minimizes the size of the tumor and permits R0 (Fig. 3). In patients with negative margins, chemotherapy should be recommended, especially where an RCC is identified or if the patient is male or aged $>65$ years. In positive-margin resection, patients will require repeated resection with brachy/boost therapy and chemotherapy (Fig. 3).

In the case of metastatic MLPS, all patients should be treated with neoadjuvant chemotherapy (3). Recurrent MLPS should be addressed with the same algorithm, but following the suggestions for the high-risk category.

The present updated algorithm will guide the surgeon towards a stepwise management plan based on clinical evidence, risk and prognostic factors for the optimization of decision making and OS. However, additional clinical trials 
are warranted to better fortify therapeutic guidelines for the treatment of MLPS. Furthermore, a meta-analysis would be a beneficial addition to the current literature, as it would allow for improved stratification of the level of evidence of the available literature. Finally, a multidisciplinary approach, including the surgeon, oncologist and radiation oncologist, is critical to optimizing an individualized treatment plan for patients with MLPS.

\section{Acknowledgements}

Not applicable.

\section{Funding}

No funding was received.

\section{Availability of data and materials}

Not applicable.

\section{Authors' contributions}

YT, AB, ASN and SS all contributed equally to the study design, data collection and analysis, and writing or reviewing of the manuscript. Data authentication is not applicable. All authors have read and approved the final manuscript.

\section{Ethics approval and consent to participate}

Not applicable.

\section{Patient consent for publication}

Not applicable.

\section{Competing interests}

The authors declare that they have no competing interests.

\section{References}

1. Conyers R, Young S and Thomas DM: Liposarcoma: Molecular genetics and therapeutics. Sarcoma 2011: 483154, 2010.

2. De Vita A, Mercatali L, Recine F, Pieri F, Riva N, Bongiovanni A Liverani C, Spadazzi C, Miserocchi G, Amadori D and Ibrahim T: Current classification, treatment options, and new perspectives in the management of adipocytic sarcomas. Onco Targets Ther 9: 6233-6246, 2016.

3. Moreau LC, Turcotte R, Ferguson P, Wunder J, Clarkson P, Masri B, Isler M, Dion N, Werier J, Ghert M, et al: Myxoid\round cell liposarcoma (MRCLS) revisited: An analysis of 418 primarily managed cases. Ann Surg Oncol 19: 1081-1088, 2012.

4. Haniball J, Sumathi VP, Kindblom LG, Abudu A, Carter SR, Tillman RM, Jeys L, Spooner D, Peake D and Grimer RJ: Prognostic factors and metastatic patterns in primary myxoid/round-cell liposarcoma. Sarcoma 2011: 538085, 2011.

5. Hoffman A, Ghadimi MP, Demicco EG, Creighton CJ, Torres K, Colombo C, Peng T, Lusby K, Ingram D, Hornick JL, et al Localized and metastatic myxoid/round cell liposarcoma: Clinical and molecular observations. Cancer 119: 1868-1877, 2013.

6. Fiore M, Grosso F, Lo Vullo S, Pennacchioli E, Stacchiotti S, Ferrari A, Collini P, Lozza L, Mariani L, Casali PG and Gronchi A: Myxoid/round cell and pleomorphic liposarcomas: Prognostic factors and survival in a series of patients treated at a single institution. Cancer 109: 2522-2531, 2007.
7. Zheng K, Yu XC, Xu M and Yang Y: Surgical outcomes and prognostic factors of myxoid liposarcoma in extremities: A retrospective study. Orthop Surg 11: 1020-1028, 2019.

8. Higgins JP, Altman DG, Gøtzsche PC, Jüni P, Moher D, Oxman AD, Savovic J, Schulz KF, Weeks L, Sterne JA, et al: The cochrane collaboration's tool for assessing risk of bias in randomised trials. BMJ 343: d5928, 2011.

9. Wells GA, Shea B, O'Connell D, Peterson J, Welch V, Losos M and Tugwell P: The Newcastle-Ottawa scale (NOS) for assessing the quality of non-randomized studies in meta-analysis, 2000.

10. Dürr HR, Rauh J, Baur-Melnyk A, Knösel T, Lindner L, Roeder F, Jansson V and Klein A: Myxoid liposarcoma: local relapse and metastatic pattern in 43 patients. BMC Cancer 18: 304, 2018

11. Wu J, Qian S and Jin L: Prognostic factors of patients with extremity myxoid liposarcomas after surgery. J Orthop Surg Res 14: 90, 2019.

12. Nishida $\mathrm{Y}$, Tsukushi S, Nakashima $\mathrm{H}$ and Ishiguro $\mathrm{N}$ : Clinicopathologic prognostic factors of pure myxoid liposarcoma of the extremities and trunk wall. Clin Orthop Relat Res 468: 3041-3046, 2010

13. Lansu J, Bovée JVMG, Braam P, van Boven H, Flucke U, Bonenkamp JJ, Miah AB, Zaidi SH, Thway K, Bruland ØS, et al: Dose reduction of preoperative radiotherapy in myxoid liposarcoma: A nonrandomized controlled trial. JAMA Oncol 7 : e205865, 2021.

14. Greto D, Saieva C, Loi M, Terziani F, Visani L, Garlatti P, Lo Russo M, Muntoni C, Becherini C, Topulli J, et al: Influence of age and subtype in outcome of operable liposarcoma. Radiol Med 124: 290-300, 2019.

15. Kilpatrick SE, Doyon J, Choong PF, Sim FH and Nascimento AG: The clinicopathologic spectrum of myxoid and round cell liposarcoma. A study of 95 cases. Cancer 77: 1450-1458, 1996.

16. Cohen HJ: Biology of aging as related to cancer. Cancer 74 (7 Suppl): S2092-S2100, 1994.

17. ten Heuvel SE, Hoekstra HJ, van Ginkel RJ, Bastiaannet E and Suurmeijer AJ: Clinicopathologic prognostic factors in myxoid liposarcoma: A retrospective study of 49 patients with long-term follow-up. Ann Surg Oncol 14: 222-229, 2007.

18. Antonescu CR, Tschernyavsky SJ, Decuseara R, Leung DH, Woodruff JM, Brennan MF, Bridge JA, Neff JR, Goldblum JR and Ladanyi M: Prognostic impact of P53 status, TLS-CHOP fusion transcript structure, and histological grade in myxoid liposarcoma: A molecular and clinicopathologic study of 82 cases. Clin Cancer Res 7: 3977-3987, 2001.

19. Smith TA, Easley KA and Goldblum JR: Myxoid/round cell liposarcoma of the extremities. A clinicopathologic study of 29 cases with particular attention to extent of round cell liposarcoma. Am J Surg Pathol 20: 171-180, 1996.

20. Callegaro D, Miceli R, Bonvalot S, Ferguson PC, Strauss DC, van Praag VVM, Levy A, Griffin AM, Hayes AJ, Stacchiotti S, et al: Development and external validation of a dynamic prognostic nomogram for primary extremity soft tissue sarcoma survivors. EClinicalMedicine 17: 100215, 2019.

21. Gustafson P: Soft tissue sarcoma: Epidemiology and prognosis in 508 patients. Acta Orthop Scand Suppl 259: 2-31, 1994.

22. Chang HR, Hajdu SI, Collin C and Brennan MF: The prognostic value of histologic subtypes in primary extremity liposarcoma. Cancer 64: 1514-1520, 1989.

23. Kilpatrick SE, Ward WG and Bos GD: The value of fine-needle aspiration biopsy in the differential diagnosis of adult myxoid sarcoma. Cancer 90: 167-177, 2000.

24. Evans HL: Liposarcoma a study of 55 cases with a reassessment of its classification. Am J Surg Pathol 3: 507-523, 1979.

25. Fiore M, Ford S, Callegaro D, Sangalli C, Colombo C, Radaelli S, Frezza AM, Renne SL, Casali PG and Gronchi A: Adequate local control in high-risk soft tissue sarcoma of the extremity treated with surgery alone at a reference centre: Should radiotherapy still be a standard? Ann Surg Oncol 25: 1536-1543, 2018.

26. Spillane AJ, Fisher C and Thomas JM: Myxoid liposarcoma-the frequency and the natural history of nonpulmonary soft tissue metastases. Ann Surg Oncol 6: 389-394, 1999.

27. Lemeur M, Mattei JC, Souteyrand P, Chagnaud C, Curvale G and Rochwerger A: Prognostic factors for the recurrence of myxoid liposarcoma: 20 cases with up to 8 years follow-up. Orthop Traumatol Surg Res 101: 103-107, 2015.

28. Bartlett EK, Curtin CE, Seier K, Qin LX, Hameed M, Yoon SS, Crago AM, Brennan MF and Singer S: Histologic subtype defines the risk and kinetics of recurrence and death for primary extremity/truncal liposarcoma. Ann Surg, Jul 5, 2019 (Epub ahead of print) 
29. Yang JC, Chang AE, Baker AR, Sindelar WF, Danforth DN, Topalian SL, DeLaney T, Glatstein E, Steinberg SM, Merino MJ and Rosenberg SA: Randomized prospective study of the benefit of adjuvant radiation therapy in the treatment of soft tissue sarcomas of the extremity. J Clin Oncol 16: 197-203, 1998.

30. Pisters P, Harrison LB, Leung D, Woodruff JM, Casper ES and Brennan MF: Long-term results of a prospective randomized trial of adjuvant brachytherapy in soft tissue sarcoma. J Clin Oncol 14: 859-868, 1996.

31. Harrison LB, Franzese F, Gaynor JJ and Brennan MF: Long-term results of a prospective randomized trial of adjuvant brachytherapy in the management of completely resected soft tissue sarcomas of the extremity and superficial trunk. Int J Radiat Oncol Biol Phys 27: 259-265, 1993.

32. Kattan MW, Leung DH and Brennan MF: Postoperative nomogram for 12-year sarcoma-specific death. J Clin Oncol 20 : 791-796, 2002 .

33. Orson GG, Sim FH, Reiman HM and Taylor WF: Liposarcoma of the musculoskeletal system. Cancer 60: 1362-1370, 1987.

34. Reitan JB, Kaalhus O, Brennhovd IO, Sager EM, Stenwig AE and Talle K: Prognostic factors in liposarcoma. Cancer 55: 2482-2490, 1985.

35. Beane JD, Yang JC, White D, Steinberg SM, Rosenberg SA and Rudloff U: Efficacy of adjuvant radiation therapy in the treatment of soft tissue sarcoma of the extremity: 20-year follow-up of a randomized prospective trial. Ann Surg Oncol 21: 2484-2489, 2014.

36. Engström K, Bergh P, Cederlund CG, Hultborn R, Willen $H$, Aman P, Kindblom LG and Meis-Kindblom JM: Irradiation of myxoid/round cell liposarcoma induces volume reduction and lipoma-like morphology. Acta Oncol 46: 838-845, 2007.

37. Knebel C, Lenze U, Pohlig F, Lenze F, Harrasser N, Suren C, Breitenbach J, Rechl H, von Eisenhart-Rothe R and Mühlhofer HML: Prognostic factors and outcome of liposarcoma patients: A retrospective evaluation over 15 years. BMC Cancer 17: 410, 2017.

38. le Grange F, Cassoni A and Seddon B: Tumour volume changes following pre-operative radiotherapy in borderline resectable limb and trunk soft tissue sarcoma. Eur J Surg Oncol 40: 394-401, 2014

39. Guadagnolo BA, Zagars GK, Ballo MT, Patel SR, Lewis VO, Benjamin RS and Pollock RE: Excellent local control rates and distinctive patterns of failure in myxoid liposarcoma treated with conservation surgery and radiotherapy. Int J Radiat Oncol Biol Phys 70: 760-765, 2008

40. Oh YJ, Yi SY, Kim KH, Cho YJ, Beum SH, Lee YH, Suh JS, Hur H, Kim KS, Kim SH, et al: Prognostic model to predict survival outcome for curatively resected liposarcoma: A multi-institutional experience. J Cancer 7: 1174-1180, 2016.

41. Muratori F, Bettini L, Frenos F, Mondanelli N, Greto D, Livi L, Franchi A, Roselli G, Scorianz M, Capanna R and Campanacci D: Myxoid liposarcoma: Prognostic factors and metastatic pattern in a series of 148 patients treated at a single institution. Int J Surg Oncol 2018: 8928706, 2018.

42. Gronchi A, Hindi N, Cruz J, Blay JY, Lopez-Pousa A, Italiano A, Alvarez R, Gutierrez A, Rincón I, Sangalli C, et al: Trabectedin and RAdiotherapy in soft tissue sarcoma (TRASTS): Results of a phase I study in myxoid liposarcoma from Spanish (GEIS), Italian (ISG), French (FSG) sarcoma groups. EClinicalMedicine 9: 35-43, 2019.

43. Aiba H, Yamada S, Mizutani J, Yamamoto N, Okamoto $\mathrm{H}$, Hayashi K, Kimura H, Takeuchi A, Miwa S, Higuchi T, et al: Preoperative evaluation of the efficacy of radio-hyperthermo-chemotherapy for soft tissue sarcoma in a case series. PLoS One 13: e0195289, 2018.

44. Vos M, Koseła-Paterczyk H, Rutkowski P, van Leenders GJLH, Normantowicz M, Lecyk A, Sleijfer S, Verhoef C and Grünhagen DJ: Differences in recurrence and survival of extremity liposarcoma subtypes. Eur J Surg Oncol 44: 1391-1397, 2018.

45. Toulmonde M, Bonvalot S, Méeus P, Stoeckle E, Riou O, Isambert N, Bompas E, Jafari M, Delcambre-Lair C, Saada E, et al: Retroperitoneal sarcomas: Patterns of care at diagnosis, prognostic factors and focus on main histological subtypes: A multicenter analysis of the French sarcoma group. Ann Oncol 25: 735-742, 2014.

46. Salduz A, Alpan B, Valiyev N, Özmen E, İribaş A, Ağaoğlu F, Bayram A, Bilgic B and Özger H: Neoadjuvant radiotherapy for myxoid liposarcomas: Oncologic outcomes and histopathologic correlations. Acta Orthop Traumatol Turc 51: 355-361, 2017.
47. Chao AH, Mayerson JL, Chandawarkar R and Scharschmidt TJ: Surgical management of soft tissue sarcomas: Extremity sarcomas. J Surg Oncol 111: 540-545, 2015.

48. Eilber FR and Eckardt J: Surgical management of soft tissue sarcomas. Semin Oncol 24: 526-533, 1997.

49. Sahu A, Sagar R, Sarkar S and Sagar S: Psychological effects of amputation: A review of studies from India. Ind Psychiatry J 25 $4-10,2016$

50. Rosenberg SA, Tepper J, Glatstein E, Costa J, Baker A, Brennan M, DeMoss EV, Seipp C, Sindelar WF, Sugarbaker P and Wesley R: The treatment of soft-tissue sarcomas of the extremities: Prospective randomized evaluations of (1) limb-sparing surgery plus radiation therapy compared with amputation and (2) the role of adjuvant chemotherapy. Ann Surg 196: 305-315, 1982.

51. Chung PW, Deheshi BM, Ferguson PC, Wunder JS, Griffin AM, Catton CN, Bell RS, White LM, Kandel RA and O'Sullivan B: Radiosensitivity translates into excellent local control in extremity myxoid liposarcoma: A comparison with other soft tissue sarcomas. Cancer 115: 3254-3261, 2009.

52. Endo M and Lin PP: Surgical margins in the management of extremity soft tissue sarcoma. Chin Clin Oncol 7: 37, 2018.

53. Enneking WF, Spanier SS and Goodman MA: A system for the surgical staging of musculoskeletal sarcoma. Clin Orthop Relat Res: 106-120, 1980.

54. Skorpil M, Rydén H, Wejde J, Lidbrink E, Brosjö O and Berglund J: The effect of radiotherapy on fat content and fatty acids in myxoid liposarcomas quantified by MRI. Magn Reson Imaging 43: 37-41, 2017

55. von Mehren M, Randall RL, Benjamin RS, Boles S, Bui MM, Ganjoo KN, George S, Gonzalez RJ, Heslin MJ, Kane JM, et al: Soft tissue sarcoma, version 2.2018, NCCN clinical practice guidelines in oncology. J Natl Compr Canc Netw 16: 536-563, 2018.

56. Pitson G, Robinson P, Wilke D, Kandel RA, White L, Griffin AM, Bell RS, Catton CN, Wunder JS and O'Sullivan B: Radiation response: An additional unique signature of myxoid liposarcoma. Int J Radiat Oncol Biol Phys 60: 522-526, 2004.

57. Chowdhry V, Goldberg S, DeLaney TF, Cote GM, Chebib I, Kim J, Lozano-Calderón SA and De Amorim Bernstein K: Myxoid liposarcoma: Treatment outcomes from chemotherapy and radiation therapy. Sarcoma 2018: 8029157, 2018.

58. Hatano H, Ogose A, Hotta T, Kawashima H, Sugita T, Sasamoto R and Endo N: Treatment of myxoid liposarcoma by marginal or intralesional resection combined with radiotherapy. Anticancer Res 23: 3045-3049, 2003.

59. Davis AM, O'Sullivan B, Turcotte R, Bell R, Catton C, Chabot P, Wunder J, Hammond A, Benk V, Kandel R, et al: Late radiation morbidity following randomization to preoperative versus postoperative radiotherapy in extremity soft tissue sarcoma. Radiother Oncol 75: 48-53, 2005.

60. Wortman JR, Tirumani SH, Tirumani H, Shinagare AB, Jagannathan JP, Hornick JL and Ramaiya NH: Neoadjuvant radiation in primary extremity liposarcoma: Correlation of MRI features with histopathology. Eur Radiol 26: 1226-1234, 2016.

61. Roberge D, Skamene T, Nahal A, Turcotte RE, Powell T and Freeman C: Radiological and pathological response following pre-operative radiotherapy for soft-tissue sarcoma. Radiother Oncol 97: 404-407, 2010.

62. de Vreeze RS, de Jong D, Haas RL, Stewart F and van Coevorden F: Effectiveness of radiotherapy in myxoid sarcomas is associated with a dense vascular pattern. Int J Radiat Oncol Biol Phys 72: 1480-1487, 2008

63. Mullen JT, Hornicek FJ, Harmon DC, Raskin KA, Chen YL, Szymonifka J, Yeap BY, Choy E, DeLaney TF and Nielsen GP: Prognostic significance of treatment-induced pathologic necrosis in extremity and truncal soft tissue sarcoma after neoadjuvant chemoradiotherapy. Cancer 120: 3676-3682, 2014.

64. Lazarev S, McGee H, Moshier E, Ru M, Demicco EG and Gupta V: Preoperative vs postoperative radiation therapy in localized soft tissue sarcoma: Nationwide patterns of care and trends in utilization. Pract Radiat Oncol 7: e507-e516, 2017.

65. Zagars GK, Ballo MT, Pisters PW, Pollock RE, Patel SR and Benjamin RS: Preoperative vs. postoperative radiation therapy for soft tissue sarcoma: A retrospective comparative evaluation of disease outcome. Int J Radiat Oncol Biol Phys 56: 482-488, 2003.

66. O'sullivan B and Davis AM: A randomized phase III trial of pre-operative compared to post-operative radiotherapy in extremity soft tissue sarcoma. Int J Radiat Oncol Biol Phys 51: $151-152,2001$. 
67. Alektiar K, Brennan M and Singer S: Local control comparison of IMRT vs. brachytherapy in primary high-grade extremity Sarcoma. Int J Radiat Oncol Biol Phys 75: S65, 2009.

68. Adjuvant chemotherapy for localised resectable soft-tissue sarcoma of adults: Meta-analysis of individual data. Sarcoma meta-analysis collaboration. Lancet 350: 1647-1654, 1997.

69. Katz D, Boonsirikamchai P, Choi H, Lazar AJ, Wang WL, Xiao L, Park MS, Ravi V, Benjamin RS and Araujo DM: Efficacy of first-line doxorubicin and ifosfamide in myxoid liposarcoma. Clin Sarcoma Res 2: 2, 2012.

70. Prestwich RJ, Taylor RE and Grimer R: Metastatic myxoid liposarcoma: Aggressive multimodality management. Clin Oncol (R Coll Radiol) 17: 130, 2005.

71. Kerr LT, Donoghue JF, Wilding AL and Johns TG: Axitinib has antiangiogenic and antitumorigenic activity in myxoid liposarcoma. Sarcoma 2016: 3484673, 2016

72. Gronchi A, Hindi N, Blay JY, RedondoA, Sanfilippo R, Morosi C, Jurado JC, Fra PL, Martinez-Trufero J, Morales CMV, et al: Trabectedin and radiotherapy in soft-tissue sarcoma (TRASTS) study: An international, prospective, phase II trial in localized myxoid liposarcoma-A collaborative Spanish (GEIS), Italian (ISG) and French (FSG) group study. J Clin Oncol 38 (15 Supppl): S11514, 2020.

73. Chen M and Kirsch DG: Safely combining trabectedin with radiotherapy to treat myxoid liposarcoma. EClinicalMedicine 9: 5-6, 2019.

74. Wakely PE Jr, Geisinger KR, Cappellari JO, Silverman JF and Frable WJ: Fine-needle aspiration cytopathology of soft tissue: Chondromyxoid and myxoid lesions. Diagn Cytopathol 12: 101-105, 1995.
75. Kilpatrick SE and Ward WG: Myxofibrosarcoma of soft tissues: Cytomorphologic analysis of a series. Diagn Cytopathol 20: 6-9, 1999.

76. Asano N, Susa M, Hosaka S, Nakayama R, Kobayashi E, Takeuchi K, Horiuchi K, Suzuki Y, Anazawa U, Mukai M, et al: Metastatic patterns of myxoid/round cell liposarcoma: A review of a 25-year experience. Sarcoma 2012: 345161, 2012.

77. Kim HS, Lee J, Yi SY, Jun HJ, Choi YL, Ahn GH, Seo SW, Lim DH, Ahn YC, Park JO and Kim SJ: Liposarcoma: Exploration of clinical prognostic factors for risk based stratification of therapy. BMC Cancer 9: 205, 2009.

78. Rydholm A and Rööser B: Surgical margins for soft-tissue sarcoma. J Bone Joint Surg Am 69: 1074-1078, 1987.

79. Trovik CS: Local recurrence of soft tissue sarcoma: A Scandinavian sarcoma group project. Acta Orthop Scand 72: $1-27,2001$.

80. Kawaguchi N, Matumoto S and Manabe J: New method of evaluating the surgical margin and safety margin for musculoskeletal sarcoma, analysed on the basis of 457 surgical cases. J Cancer Res Clin Oncol 121: 555-563, 1995. 